\section{Neuropsychobiology}

Aoki, Y. 34

Asakawa, T. 34

Canuet, L. 34

Dierks, T. 6

Hata, M. 34

Hayashi, T. 34

Hirata, K. 5, 25, 49

Iijima, M. 17

Ikeda, S. 34

Inoue, Y. 25

Ishii, R. 34

Iwase, M. 34
Jann, K. 6

Katsimichas, T. 34

Kimoto, K. 49

Kinoshita, T. 5, 6

Koenig, T. 6

Mase, R. 17

Mizuno-Matsumoto, Y. 34

Morita, A. 42

Nishida, K. 6

Okamoto, E. 34

Osawa, M. 17
Pascual-Marqui, R.D. 34

Razavi, N. 6

Sasai, T. 25

Shimizu, S. 17

Takashima, R. 49

Takeda, M. 34

Tanaka, H. 49

Uchiyama, S. 17

Watanabe, Y. 49

Yoshimura, M. 6

\title{
Subject Index Vol. 71, No. 1, 2015
}

$\alpha$ band 34

Beta power 17

Chronic hepatitis C 42

Electroencephalography 6, 25, 34, 49 Emotion 34

Encephalopathy 42

Event-related desynchronization 17

Exact low-resolution brain electromagnetic tomography 34

Functional connectivity 34

- magnetic resonance imaging 6
Gamma power 17

Gravity center 49

Idiopathic form 25

Interferon 42

Migraine 49

Mild cognitive impairment 25

Neurophysiology 6

Neuroticism 34

Photic driving 49

Power spectral analysis 25
Quantitative electroencephalography 42

REM 25

Resting state networks 6

Sleep behavior disorder 25

$\alpha$-Synucleinopathies 25

Time-frequency analysis 17

Topography 49

Visual go/no-go paradigm 17 